EGU2020-5709, updated on 22 Feb 2021

https://doi.org/10.5194/egusphere-egu2020-5709

EGU General Assembly 2020

(c) Author(s) 2021. This work is distributed under

the Creative Commons Attribution 4.0 License.

\title{
Uncertainties associated with the delineation of management zones in precision agriculture
}

\author{
Tomás R. Tenreiro ${ }^{1}$, Margarita García-Vila ${ }^{2}$, José A. Gómez ${ }^{1}$, and Elías Fereres ${ }^{1,2}$ \\ ${ }^{1}$ Spanish Council for Scientific Research - Institute for Sustainable Agriculture, Córdoba, Spain \\ (roquettetenreiro@gmail.com) \\ ${ }^{2}$ Department of Agronomy, University of Córdoba, Córdoba, Spain
}

The characterization of spatial variations in soil properties and crop performance within precision agriculture, and particularly the delineation of management zones (MZ) and sampling schemes, are complex assignments currently far from being resolved. Considerable advances have been achieved regarding the analysis of spatial data, but less attention has been devoted to assess the temporal asymmetry associated with variable crop xyear interactions. In this case-study of a 9 ha field located in Spain, we captured interactions between both spatial and temporal variations for two contrasting seasons of remotely sensed crop data (NDVI) combined with several geomorphological properties (i.e., elevation, slope orientation, soil apparent electrical conductivity - ECa, \%Clay, \%Sand, pH). We developed an algorithm combining Principal Component Analysis (PCA) and clustering k-means and succeeded to delineate four MZ's with a satisfactory fragmentation degree, each one associated with a different Elevation $\times E C a \times N D V I$ combination. Simulated yield maps were generated using NDVI maps correlated to ground cover to establish initial conditions in simulation settings with a crop model. Yield maps were spatially correlated but fitted into variograms with irregular spatial structure. Both CV and spatial patterns did not show consistency from year to year. The results indicate that MZ's temporal instability is an important issue for site-specific management as agronomic implications varied greatly with crop $\times y e a r$ setting. We observed differences, not only regarding NDVI patterns but also in yield response to the combination of Elevation $\times E C a$ (and Texture) depending on the seasonal rainfall. A reduction of $14 \%$ of the 'Goodness of Variance Fit' was observed for simulated yield from the first to the second cropxyear, highlighting the difficulties in the delineation of MZ's with persistent confidence. The interpretation of $M Z \times Y$ ield associations was not straight forward from the metrics selected here as it also depended on agronomic knowledge. We believe that precision agriculture will benefit greatly from improved protocols for $\mathrm{MZ}$ delineation and sampling schemes. However, the uncertainty associated with temporal asymmetry of yield clustering and MZ's interpretation reveals that 'automated digital agricultural systems' are still far from reality. 